\title{
Editorial: Contexts and controversy
}

\section{Heather Joshi}

The research reported in this issue of the journal deals with a range of aspects of the life course, sometimes controversial. The topics concern moving home in childhood, having a child as a teenager and how life transitions vary by disability in adolescence and by sexuality reported in later life. Taken together, these contributions bring out the need to appreciate their contrasting contexts.

The first two papers add to a debate about the consequences of residential mobility for children's educational achievements. Sergei Vidal and Janeen Baxter look at outcomes in children around age 12 in 2010, the older cohort in the Longitudinal Study of Australian Children. The authors conclude that having moved home in the preceding years is not necessarily disadvantageous in itself, although some moves do accompany or reflect adverse events or circumstances. A similar conclusion was reached for pre-school children in the UK Millennium Cohort and US Fragile Families and Child Wellbeing Study in the early years of the present century, in the special issue of Longitudinal and Life Course Studies, July 2016. However the second paper in the current issue, by Juli Simon Thomas, puts childhood residential mobility more squarely in the category of disruptive events, along with family break-up and parental job loss, as leading to poorer educational outcomes. Her evidence covers a longer period, 1968 to 2011, from the Panel Study of Income Dynamics (PSID) in the USA, and focuses on the educational transitions at later stages: finishing high school, starting college and completing college. There are several explanations for the apparent contradiction, which readers may like to explore: consequences of mobility may vary by age of assessment, or age at mobility, outcomes of moves may be affected by the nature of their destinations, and, most importantly, by the policy context of cash and housing support for families and for the financing of college education. The debate is not closed.

The study by Gina Erickson and Ross Macmillan in the third article focuses on disability as a possible determinant of transitions in early adulthood. There is no evidence about disability in the first two papers, but it was a major focus of the US National Longitudinal Study of Adolescent Health (AdHealth) used here. Parents reported sufficient information to classify young people by disabilities in childhood as physical, cognitive (abnormally low vocabulary score) and (diagnosed) learning difficulties. The authors invest effort in characterising early adult transitions through education, employment, partnership and parenthood. They use latent class analysis to identify seven pathways for males and females separately, which are of interest in themselves. Physical disability was, unexpectedly, not found to be particularly predictive of taking a slow track through these milestones, but low cognitive functioning impeded educational, and consequently other, progress. In other studies where disability information is not available (such as Simon Thomas, this issue), low cognitive ability may underlie low educational attainment.

Two contributions deal with the transition to parenthood as a teenager, where opinion divides as to whether adverse outcomes for parent and child are due to premature childbearing itself or selection into it on pre-existing disadvantages. Both use whole population register data from Scandinavia, a region known for particularly low teen parenthood, but where there is nevertheless concern about its consequences. Sara Kalucza draws her evidence from people born over a five-year period in Sweden (1989-94), reaching 20 in 2009-2014. The inclusion of men as well as women who become parents before age 20 is novel. The study asks how far the record of mental ill-health in childhood is merely a facet of the social disadvantage associated with teen parenthood. Mental health problems, in girls and boys, were found to play an independent role. This suggests interventions to raise confidence in alternative opportunities to parenthood among young people with mental problems. The indicator of mental ill-health was derived from the register on prescription of psychotropic drugs. These are likely to overlap with the 'learning disabilities' used in the AdHealth study (Erickson and Macmillan, this issue), but these parent-reported diagnoses did not predict early parenthood in the US study. This international comparison should be made cautiously, if only because of the different contexts in which child mental health is treated. Heini Väisänen's Research Note on teenage pregnancy in 
Finland relates it to what follows in educational attainment by age 30 . This study is confined to women, but here the novelty is that the register offers important evidence on teenage pregnancies that end in abortion, not easily collected in surveys. The women with abortions had intermediate educational outcomes between the teen mothers and those who were not pregnant as teenagers. This suggests some social selectivity into teenage pregnancy. The cohort studied was born 1975-79, and thus passed through teenage years in the 1990s. The author notes that the picture may have changed in Finland since then, and suggests that the social selectivity of teenage motherhood may be greater in less accommodating welfare states such as the USA. Although not directly comparable, the estimated covariates of the school-to-family trajectory for women in Erickson and Macmillan's table 3 provides some evidence consistent with this, in the inverse association of a young woman's entry to early motherhood with her own parents' educational level.

The paper by Dylan Kneale and Robert French extends to a larger life course canvas up to retirement and old age. The information comes from people who were at least 50 years old in 2006, reporting their life histories to the English Longitudinal Study of Ageing (ELSA). It covers people living in England who had been born up to
1946, mainly cohorts experiencing early adulthood in the 1950s and early 1960s. The novelty here is that the respondents are classified by sexuality on the basis of responses given to ELSA (in 2012), on whether they had ever (onset unspecified) practised or desired same-sex relations. From a range of possible definitions, $4 \%$ of respondents ( $n$ 159) were estimated to be either Lesbian, Gay or Bisexual (LGB). The life transitions and health of these survivors were on the whole not dissimilar to those of heterosexual informants. They were neither more nor less likely to enter a parental role early, but less likely to do so eventually ( $71 \%$ versus 91\%). Using event history techniques, a few other lifetime turning points (among those measured) stood out as differentiating LBG histories. They were more likely to have taken on a caring role for a friend or family member, or experienced sexual assault and at an earlier point than the heterosexual respondents. Much of the time recoded here spanned the period when male homosexual practices were illegal. It also covered the period of mortality from AIDS, and stops before gay marriage became a possibility. Here too conclusions need to be tempered by context. Nevertheless this pioneering effort, along with the other papers in this issue, points to questions that still need to be asked. 